\title{
A relationship between claudication of the cauda equina and the small aorta syndrome
}

\author{
KR POSKITT, GD PERKIN, RM GREENHALGH \\ From the Departments of Neurology and Surgery, Charing Cross Hospital, London
}

SUMMARY Two cases of claudication of the cauda equina due to ischaemia secondary to distal aortic disease in the Small Aorta syndrome are reported. The association has not been previously reported and the appropriate management is discussed.

The Small Aorta syndrome is now generally recognised as an established clinical entity, ${ }^{1-6}$ in which the arterial tree has a characteristic appearance. The infra-renal aorta tapers down in size so that the aortic bifurcation and the vessels below it are relatively hypoplastic. Atherosclerotic changes occur in the lower aorta but the vessels below the iliacs, apart from being narrow, are relatively disease free. It has been described in females with a relatively early age of onset who are below average height and have smoked an average of 20 cigarettes per day for 20 years or more. ${ }^{2}$ The patients are not usually overweight and biochemical investigations generally demonstrate hyperlipidaemic abnormalities, principally type $11 \mathrm{a}^{2}$ or type IV. ${ }^{3-5}$

Previously, the typical clinical presentation of this syndrome has been with bilateral buttock, thigh and calf claudication. We describe two patients with this syndrome whose presentation suggested a diagnosis of claudication of the cauda equina. The symptoms were totally relieved by surgical bypass of the aortic obstruction.

\section{Case reports}

Case 1 This 61-year-old lady presented with a complaint of severe low back pain and pain radiating down the back of the legs into the soles of her feet when she walked 50 yards on the flat. If she tried walking further she lost sensation in the soles of her feet and was less aware of contact with the ground. With rest, a sensation of cold water down the back of her legs was followed by the return of normal sensation. At the age of $56 \mathrm{yr}$, she had complained of severe backache, the cause of which had not been established. She did not complain of claudication in the buttocks, thighs or calves and had no history of ischaemic rest pain in the toes. She had

Address for reprint requests: Mr KR Poskitt, Department of Surgery, Charing Cross Hospital, Fulham Palace Road, London W6 8RF, UK.

Received 6 April 1984 and in revised form 14 June 1984. Accepted 16 June 1984. no sphincter disturbances and no history of back trauma. She had smoked 15 cigarettes per day for 35 years.

Examination revealed a $5 \mathrm{ft} 4 \mathrm{in}(160 \mathrm{~cm})$ slim lady who had a blood pressure of $160 / 95 \mathrm{mmHg}$. A systolic bruit was heard in the abdomen. Both femoral pulses were just palpable with no pulses below this. Examination of the skeletal system showed no abnormality and the only positive finding in the examination of the nervous system was that straight leg raising was slightly limited, producing pain. The left and right ankle Doppler pressures were reduced at $125 \mathrm{mmHg}$ with reference to a brachial pressure of $190 \mathrm{mmHg}$ and the femoral arterial waveforms were damped. The serum cholesterol and triglyceride estimations were both within normal limits. A myelogram was normal. An arteriogram was performed with some difficulty and the films, of poor quality, failed to show a specific lesion.

In view of the severe symptoms, the clinical findings and results of the non-invasive Doppler pressure and waveform analysis, it was decided that the cause of her symptoms was due to claudication of the cauda equina caused by aortic disease and surgery was advised. At operation, the aorta was seen to taper down in size from the level of the renal arteries to the bifurcation. Just proximal to the bifurcation, there was marked localised stenosing arterial disease. A local endarterectomy was performed in the upper infra-renal aorta to facilitate the insertion of an aorto-bi-external iliac Dacron graft to normal external iliac arteries. In the three and a half years of follow up, she has been asymptomatic with bilateral ankle Doppler pressures of $175 \mathrm{mmHg}$ with reference to a brachial systolic pressure of $145 \mathrm{mmHg}$. The ankle pressures now rise after a period of exercise on a treadmill at $4 \mathrm{~km}$ per hour on a $10 \%$ slope for 1 minute. This indicates that there is no haemodynamically significant stenosing lesion in the arteries supplying the lower limbs. ${ }^{7}$

Case 2 This 31-year-old lady originally presented with paraesthesiae in the buttocks radiating down the back of each leg into the soles of the feet. These symptoms occurred only when she walked 800 yards on the flat and were followed by a heaviness and numbness in both legs subsiding with rest after 3 minutes. The symiptoms had started during her pregnancy two and a half years previously, and were worsening. She did not complain of buttock, thigh or calf claudication and had no backache, rest pain or recent 


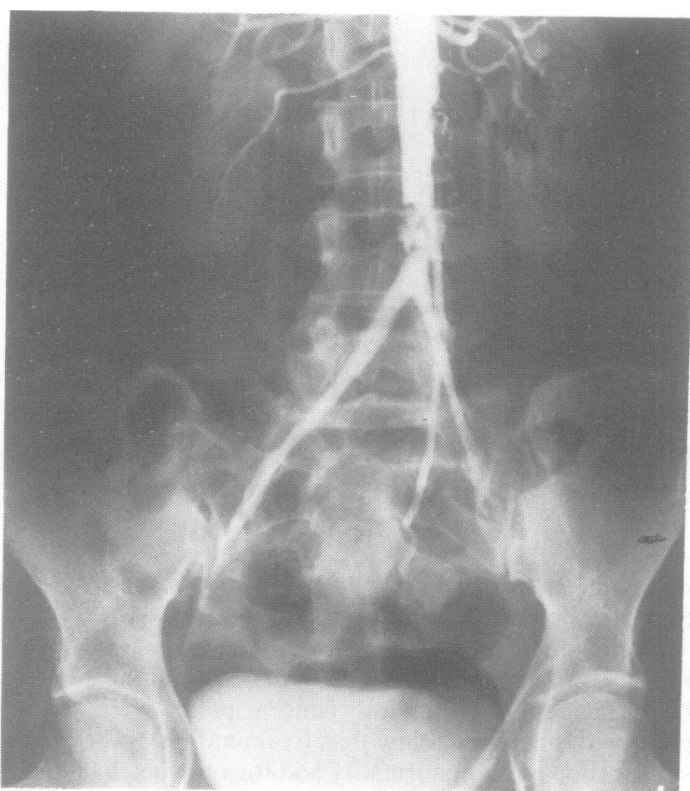

Fig 1 Angiogram of case (2) demonstrating the infrarenal aorta tapering in size with a $90 \%$ stenosis just proximal to the aortic bifurcation.

claudication and had no backache, rest pain or recent trauma. She had smoked 20 or more cigarettes per day for 12 years and had been on a contraceptive pill for 18 months. In the past, she had developed pre-eclamptic toxaemia in two of her pregnancies and had suffered one miscarriage.

Examination revealed that she was slim and $4 \mathrm{ft} 11$ ins $(147 \mathrm{~cm})$ tall. She was normotensive and had a systolic bruit in the supra-umbilical region. She had absent pulses in the lower limbs, but all other pulses were normal with no bruits. Skeletal system and nervous system examination were completely normal. Doppler studies showed bilateral ankle pressures of $70 \mathrm{mmHg}$ with a brachial pressure of $140 \mathrm{mmHg}$. Both femoral artery waveforms were markedly damped. Serum triglycerides were $1.9 \mathrm{mmol} / 1$ (normal 0.5 to $2.2 \mathrm{mmol} / \mathrm{l}$ ) and the serum cholesterol was $7.4 \mathrm{mmol} / \mathrm{l}$ (normal 3.4 to $6.5 \mathrm{mmol} / \mathrm{l}$ ). Arteriography (fig 1) showed tapering of the lower abdominal aorta with a $90 \%$ stenosis just proximal to the bifurcation. The lumbar arteries were occluded and the distal vessels hypoplastic, but smooth and free from disease. At operation, the arteriographic findings were confirmed. A local endarterectomy in the upper intrarenal aorta was performed to facilitate insertion of an end-to-side aorto-bi-external iliac Dacron graft. After the operation, the resting Doppler pressures in both ankles were normal and showed the normal rise following a period of exercise. She was completely symptom free 12 months after surgery.

\section{Discussion}

Symptomatic peripheral vascular disease predominantly affects the male population. The incidence of peripheral vascular disease in young females is partic- ularly low and may result from hormonal protection. $\bar{z}$ In recent years a group of females who present at an $\mathbb{D}$ early age with symptomatic aorto-iliac disease have been described.

In 1969, Johnson" suggested the term "Small Blood Vessel Syndrome" for young females presenting with symptomatic occlusive aorto-iliac disease. He noted that they were below average height, had mild truncal obesity, and had significant tapering of the infra-renal terminal aorta demonstrated by angiography. Greenhalgh and Taylor ${ }^{1}$ described a group of 27 females with stenosing arterial disease confined to the terminal $\bar{\tau}$ abdominal aorta, which tapered down in size to its bifurcation. He called these "little women with blocked aortas", but later ${ }^{2}$ Greenhalgh adopted the term "Small Aorta syndrome". Females in this group were young (mean age $49 \mathrm{yr}$ ) and normal endocrinologically and were noted to be below average height but not obese as reported in Johnson's series. ${ }^{6}$ They had all been smokers for many years. Seventy eight percent had abnormal lipoprotein patterns with $70 \%$ having elevated serum cholesterol levels. He drew attention to the curious arteriographic finding of localised disease only at one site in the descending arterial tree. The peripheral arteries were on the whole $\overline{5}$ ot small but completely disease free in other sites although $30 \%$ had already had a myocardia infarction even at that early age. He felt that this was $\vec{c}$ a recognisable syndrome. All 27 cases reported hare severe symptoms of bilateral buttock, thigh and cal claudication and all required surgical correction. In tially, the surgical procedure performed involved $\$$ local endarterectomy and the insertion of a Dacro patch fashioned in the shape of an inverted Y. Occasionally, the disease extended down the iliac vessels and in these patients a bifurcated Dacron graft to the external iliac arteries was inserted. This became the procedure of choice in preference to endarterectomy and patching due to the tendency for recurrent stenosis to occur in the latter, and has been a most satisfactory procedure.

Lipid abnormalities are common in young women presenting with aorto-iliac disease. ${ }^{35}$ These patients appeared to have an arteriographically hypoplastic lower aorta. A profile using specific apolipoprotein ratios is said to distinguish patients with the Small Aorta syndrome from those with more diffuse and segmented arterial disease referred to as the "Stenosing Peripheral Arterial Disease" group. ${ }^{8}$

There are several risk factors that lead to the development of arterial disease in young women. It appears that the Small Aorta syndrome occurs in patients who have a preponderance of these. Arnot and Louw ${ }^{9}$ suggest that a smaller diameter lower abdominal aorta is due to a congenital defect in which there is excessive fusion of the embryonic dorsal aortas around the 25th 
day of intrauterine life. Females in whom this occurs appear to have a higher risk of developing lower aortic occlusive disease. In addition, if they are small in stature, smoke for many years and suffer from a hyperlipidaemic state, they are likely to develop symptomatic occlusive aorto-iliac disease at an early age.

We believe both cases described fall into the category of the Small Aorta syndrome. Case 1 initially presented at the age of 56 years, was small in stature and had smoked heavily for 35 years. She however did not show the abnormal lipoprotein pattern which is present in up to $78 \%$ of patients, but did show the significant tapering of the lower abdominal aorta. Case 2 was very young at presentation, had smoked heavily for many years and was $4 \mathrm{ft} 11$ ins $(147 \mathrm{~cm})$ tall. She had an elevated cholesterol level and a marked tapering of her lower abdominal aorta with a $90 \%$ stenosis. She had developed pre-eclamptic toxaemia during two of her pregnancies and had had a miscarriage. Clemetson ${ }^{10}$ has noted the association of hypoplasia of the distal aorta with eclampsia and recurrent intra-uterine foetal death.

The anterior two-thirds of the spinal cord is supplied by the anterior median spinal artery which in turn receives a contribution from as many as $17 \mathrm{seg}$ mental arteries. The largest of these, the great anterior medullary artery usually enters the spinal canal at the first or second lumbar segment and then turns upwards. ${ }^{11}$ Four lumbar arteries arising from the aorta opposite the upper four lumbar vertebrae and an ilio-lumbar artery arising from the posterior division of the internal iliac artery produce posterior segmental arteries which in turn supply spinal branches entering at the intervertebral foramina.

Claudication of the spinal cord was first described by Dejerine. ${ }^{12}$ Later, Reichert et al $^{13}$ described four cases of spinal cord claudication and supported their hypothesis as to its causation with animal experiments, in which they ligated one or more lumbar arteries in dogs and produced weakness and delayed spasticity in the ipsilateral hind limb. A more recent study by Bergmark ${ }^{14}$ summarised Dejerine's cases, and added a further two patients. The condition is rare and has never been fully defined. It seems to be characterised by paroxysmal lower limb weakness induced by exercise and relieved by rest. The peripheral pulses are normal and it is thought the condition is secondary to disease of the spinal arteries. The distribution of arterial disease in the two patients reported was not likely to have affected the great anterior medullary artery, nor was their clinical picture typical of this condition.

Intermittent claudication of the cauda equina has been described in two forms, a postural type induced by prolonged standing and an ischaemic form precipitated by walking. ${ }^{15}$ The condition predominates in men. Radiological abnormalities consist of protrusion of one or more lumbar discs against the laminae or a bulging ligamentum flavum. It has been suggested that the exercise induced symptoms are the result of compression of confined nerve roots by their dilating arteries during exercise. ${ }^{16}$ Experimental evidence postulating a relative ischaemia of the cauda equina during exercise in such patients is open to criticism. ${ }^{17}$ Patients with claudication of the cauda equina develop pain and paraesthesiae which begin in the feet and extend upwards, sometimes to the thigh and buttocks. The reverse spread may occur. With cessation of walking, or sitting, symptoms recover, usually within 3 minutes. A small proportion of patients develop limb weakness but neurological signs are often not conspicuous, though they may appear with exercise. Both the patients reported showed this symptom complex. In neither was there evidence suggesting lumbar canal stenosis or central disc protrusion. One patient had a normal myelogram and in the other, symptoms were permanently relieved by arterial surgery.

The Leriche syndrome occurs in males and follows terminal aortic or bifurcation obstruction. It is characterised by lower limb wasting, impotence and severe muscle pain in the buttocks, thighs and calves during exercise. Some patients describe paraesthesiae rather than pain but typically these appear in all the affected areas simultaneously rather than showing the spread seen in neurogenic claudication. Gilfillan et al ${ }^{18}$ suggested that the pain in these ischaemic cases follows a myotomal distribution, that it correlates very closely with the amount of muscle exercise, and that it subsides very rapidly with rest.

They reported four patients in whom certain features had suggested neurogenic claudication despite the findings of vascular obstruction. None of the cases was operated upon, so that a definitive association of the symptoms with the vascular disease could not be made. Two of the patients had had normal myelograms and one had had an explorative laminectomy with negative results. All four cases described both paroxysmal pain and numbness with exercise, the numbness sometimes appearing in the perineum or sometimes in the distal part of the lower limbs. Despite these sensory symptoms, the authors assumed that the patients' symptoms were related to ischaemia of muscle and did not consider the possibility that in some instances, there might be critical impairment of flow to the cauda equina and nerve roots.

We believe that in both of our patients, critical impairment of blood flow to the cauda equina and its associated nerve roots occurred during exercise and became manifest as a clinical syndrome indistinguishable from intermittent claudication of the cauda 

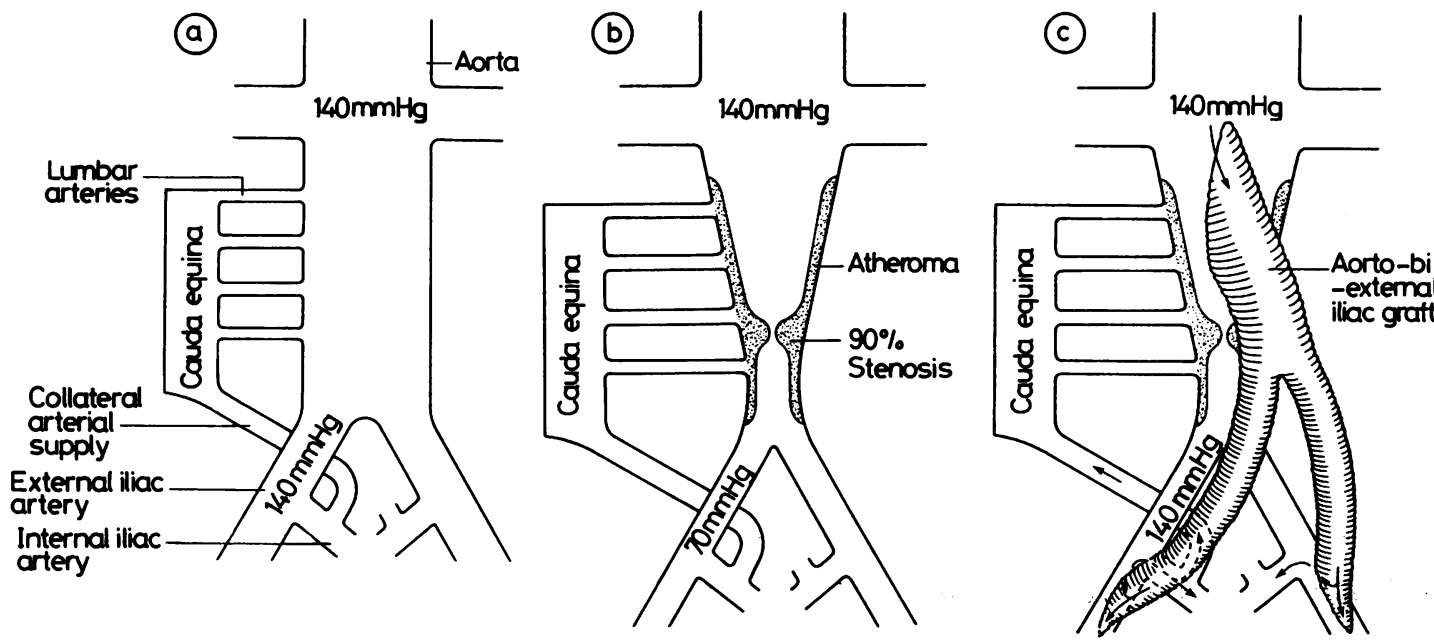

Fig 2(a) Angiogram showing a normal arterial supply to the cauda equina from the lumbar arteries and collaterals from the iliac system. (b) demonstrates the findings in case 2. The lumbar arteries are occluded and the tapered aorta has a $90 \%$ stenosis. The perfusion pressure from the iliac system is $70 \mathrm{mmHg}$ (50\% of the normal perfusion pressure). (c) demonstrates case 2 following bypass grafting, the perfusion pressure is restored to $140 \mathrm{mmHg}$ and the cauda equina is supplied by collaterals from the iliac system.

equina. The relationship of these symptoms to exercise is probably the consequence of increased blood flow to the lower limbs, stealing further circulation from the lumbar and ilio-lumbar arteries. Insertion of an aorto-bi-external iliac graft proved effective in improving perfusion to the cauda equina by retrograde flow via the graft and the ilio-lumbar artery (fig $2, \mathrm{a}, \mathrm{b}, \mathrm{c})$.

This concept of the causation of symptoms in distal aortic obstruction has not been previously suggested. Kendall and Andrew ${ }^{19}$ recorded a patient with angiographically proven steal from the anterior spinal artery to the aorta below a previously incompletely corrected coarctation of the thoracic aorta. The patient complained of paroxysmal weakness with exercise, confirmed on examination, and the case most closely resembles claudication of the spinal cord.

The typical presentation of the Small Aorta syndrome with bilateral buttock, thigh and calf claudication normally leads to the patient being assessed by a vascular surgeon. We have demonstrated that certain cases of the Small Aorta syndrome present with neurological symptoms indistinguishable from those of neurogenic claudication of the cauda equina. Patients, especially females, presenting in this way, require careful assessment of their vascular tree as well as their lumbar canal and may ultimately require the same arterial reconstruction used for those cases presenting with buttock, thigh and calf claudication.

\section{References}

${ }^{1}$ Greenhalgh RM, Taylor GW. Little women with blocked aortas. Br J Surg 1974;61:923-4.

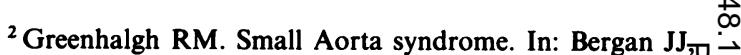
Yao JST eds: Surgery of The Aorta and its Branches New York; Grune \& Strattoǹ Inc. 1979. 183-90.

${ }^{3}$ De Laurentis DA, Friedmann P, Wolferth CC, Wilson A Naide D. Atherosclerosis and the hypoplastic aorto-iliae system. Surgery 1978;83:27-37.

${ }^{4}$ Cronenwett JL, Davis T, Gooch JB, Garrett HE. Aortoe iliac occlusive disease in women. Surgery 1980\% 88:775-84.

${ }^{5}$ Holmes DR, Burnank MK, Fulton RE, Bermatz PEêे Arteriosclerosis obliterans in young women. $\mathrm{Am} \mathrm{J} \mathrm{Med+}$ 1979;66:997-1000.

6 Johnson TE. Small Blood vessel syndrome. Minn Mid 1969;52:1903-5.

${ }^{7}$ Laing SP, Greenhalgh RM. Standard exercise test to assess peripheral arterial disease. $\mathrm{Br}$ Med $J$ 1980;280:13-16.

${ }^{8}$ McConathy MJ, Greenhalgh RM, Alaupovic P et al. Plasma lipid and apolipoprotein profiles of women with two types of peripheral arterial disease. Atherosclerosis 1984;50:295-306.

${ }^{9}$ Arnot RS, Louw JH. The anatomy of the posterior wall of the aorta. $S$ Afr Med J 1973;47:899.

${ }^{10}$ Clemetson CAB. Aortic Hypoplasia and its significance in the aetiology of pre-eclamptic toxaemia. $\mathrm{Br} J$ Obstet Gynaecol 1960;67:90.

${ }^{11}$ Gillilan LA. The arterial blood supply of the human spinal cord. J Comp Neurol 1958;110:75-100.

${ }^{12}$ Dejerine J. La Claudication Intermittente De La Moelle Epinière. Presse Medicale 1911;19:981-4.

${ }^{13}$ Reichert FL, Rytand DA, Bruck EL. Atherosclerosis of the lumbar segmental arteries producing ischaemia of the spinal cord and consequent claudication of the thighs. Am J Med Sci 1934;197:792-806.

14 Bergmark G. Intermittent spinal claudication. Acta Med Scand Supp 1950;246:30-6. 
${ }^{15}$ Wilson CB. Significance of the small lumbar spinal canal; cauda equina compression syndromes due to spondylosis. Part 3: Intermittent Claudication. J Neurosurg 1969;31:499-505.

${ }^{16}$ Blau JN, Logue V. Intermittent claudication of the cauda equina. An unusual syndrome resulting from central protrusion of a lumbar intervertebral disc. Lancet 1961;1:1081-6.

${ }^{17}$ Macleish DG. Claudication of the cauda equina. Aust NZ
J Surg 1972;47:62-4.

${ }^{18}$ Gilfillan RS, Jones OW Jnr, Roland SI, Wylie EJ. Arterial occlusions simulating neurological disorders of the lower limbs. JAMA 1954;154:1149-52.

${ }^{19}$ Kendall BE, Andrew.J; Neứogenic intermittent claudication associated with aortic steal from the anterior spinal artery complicating coarctation of the aorta. $J$ Neurosurg 1972;37:89-94. 